\title{
Silent Giant Left Atrium: A Case Report
}

Shweta Vohra ${ }^{1}$, Akshyaya Pradhan ${ }^{2}$, Vikash Jaiswal ${ }^{3}$, Prachi Sharma ${ }^{2}$, Nishan Pokhrel ${ }^{4}$, and David Song 5

${ }^{1}$ King George's Medical University

${ }^{2}$ King George Medical College

${ }^{3}$ AMA School of Medicine

${ }^{4}$ Tribhuvan University Institute of Medicine

${ }^{5}$ Icahn School of Medicine at Mount Sinai Elmhurst Hospital

November 20, 2021

\begin{abstract}
We report a case of a 34-year-old female with low-grade dyspnea on exertion despite markedly dilated left atrium (LA) on chest radiography which clouded our interpretation of the cause of enlarged cardiac silhouette. However, echocardiogram proved useful in demonstrating severe mitral stenosis and the giant LA.
\end{abstract}

TITLE: Silent Giant Left Atrium: A Case Report

\section{AUTHORS}

${ }^{1}$ Shweta Vohra : (shawetamedicine@gmail.com), (Department of Cardiology), (King George's Medical University, Lucknow, India)

2 Akshyaya Pradhan: (akshyaya33@gmail.com), (Department of Cardiology), (King George's Medical University, Lucknow, India)

3 Vikash Jaiswal: (vikash29jaxy@gmail.com) (Larkin Community Hospital, South Miami, Florida, USA) (ORCID ID: https://orcid.org/0000-0002-2021-1660 )

${ }^{4}$ Prachi Sharma: (drprachi1009@gmail.com), (Department of Cardiology), (King George's Medical University, Lucknow, India)

${ }^{5}$ Nishan Babu Pokhrel: (nishanpokhrel1@iom.edu.np) (Department of Internal Medicine, Tribhuvan University Institute of Medicine, Kathmandu, Nepal) (ORCID ID:https://orcid.org/0000-0002-4278-5753 )

${ }^{6}$ David Song: (xdavidsong@gmail.com ) (Department of Internal Medicine, Icahn School of Medicine at Mount Sinai Elmhurst Hospital, Queens, NY, USA)

\section{CORRESPONDING AUTHOR}

Nishan Babu Pokhrel

Department of Internal Medicine

Tribhuvan University Institute of Medicine

Kathmandu, Nepal

nishanpokhrel1@iom.edu.np 


\section{ABSTRACT \\ INTRODUCTION}

Enlarged left atrium (LA) is a common finding in severe mitral stenosis (MS) and severe mitral regurgitation. It typically presents with dyspnea in proportion to the enlarged atrium. There is a poor correlation between the size of the left atrium and the severity of the MS. Our case creates clinicians' awareness to consider full diagnostic workup for enlarged LA as patients may not always have symptoms.

We report a case of a 34-year-old female with low-grade dyspnea on exertion despite markedly dilated left atrium (LA) on chest radiography which clouded our interpretation of the cause of enlarged cardiac silhouette. However, echocardiogram proved useful in demonstrating severe mitral stenosis and the giant LA.

\section{KEY CLINICAL MESSAGE}

The use of chest X-ray, echocardiogram and computed tomography to evaluate for giant cardiomegaly in asymptomatic patients with a history of rheumatic heart disease can help evaluate and prevent unnecessary invasive procedures.

\section{KEYWORDS}

Rheumatic heart disease, Mitral stenosis, Echocardiography

\section{ABBREVIATIONS}

$\mathrm{RHD}=$ Rheumatic Heart Disease, $\mathrm{MS}=$ Mitral Stenosis, $\mathrm{MR}=$ Mitral Regurgitation, LA= Left Atrium, $\mathrm{MVR}=$ Mitral Valve Replacement

\section{INTRODUCTION}

Enlarged left atrium (LA) is a common finding in severe mitral stenosis (MS) and severe mitral regurgitation (MR). It can expand to giant proportions. The unusual finding in our thirty-year-old female presenting with only mild dyspnea despite markedly dilated LA prompted us to report this case. The chamber was so enormous that it was challenging to interpret the cause of the enlarged cardiac silhouette on the chest radiography.

\section{CASE PRESENTATION}

A thirty-year-old female with no significant medical history presented with dyspnea on exertion (NYHA class I). The physical examination revealed a blood pressure of $110 / 70 \mathrm{~mm} \mathrm{Hg}$ with an irregular pulse rate of 76 beats/minute and had an oxygen saturation of $98 \%$ on room air. The neck veins were not distended. The lungs were clear to percussion and auscultation. The examination of the heart revealed a mid-diastolic as well as grade III pansystolic murmur at the apex. The abdominal examination yielded normal findings, and there was no peripheral edema. The electrocardiogram on admission showed atrial flutter with a variable block (Figure 1). The chest radiography revealed massive cardiomegaly with the left heart border showing a prominent main pulmonary artery and left atrial appendage. The right heart border showed a double contour of atrial shadow (Figure 2). An echocardiogram showed a giant left atrium measuring $14.4 \mathrm{~cm}$ x $16.7 \mathrm{~cm}$ that encroached on the other cardiac chambers with a left ventricular ejection fraction of $48 \%$ (Figure 3). Thickened mitral valves along with doming and restricted motion of anterior and posterior mitral leaflets were diagnostic of rheumatic heart disease (RHD). There was severe mitral stenosis with a mitral valve area of $0.9 \mathrm{~cm}^{2}$ on mitral valve planimetry and moderate MR with vena contracta of $4 \mathrm{~mm}$ and an effective regurgitant orifice area of $0.3 \mathrm{~cm}^{2}$. The patient was administered furosemide $20 \mathrm{mg}$ PO daily with improvement and was discharged from the hospital. She reported continued improvement in her dyspnea on her follow-up visit in the outpatient clinic two weeks later.

\section{DISCUSSION}


LA size greater than $6.5 \mathrm{~cm}$ is considered as giant LA [1]. Giant LA can be caused by numerous etiologies shown in Table 1. The most common etiology is from long-standing rheumatic mitral valve regurgitation or mixed mitral valve disease with predominant regurgitation [2]. The exact pathophysiology causing giant LA, only in a subset of RHD patients, is not well understood. However, two factors that may contribute are increased LA pressure and weakening of LA wall by rheumatic pancarditis [2,3]. When mitral valve disease is a long-standing process, it leads to LA enlargement as a compensatory mechanism to ease the markedly increased LA pressure which could lead to pulmonary congestion due to back-pressure hemodynamics, hence protecting the lung from the development of pulmonary hypertension [4]. While these compensatory mechanisms help the patient remain asymptomatic for a long time, he or she gradually deteriorates due to progressive increase in LA pressure leading to an inevitable increase in pulmonary venous pressure.[3]. LA enlargement can lead to the development of atrial fibrillation which can lead to further enlargement of the LA [5]. Giant LA can lead to various complications including the increased propensity of thrombus formation and thromboembolic events, pulmonary edema, and pulmonary hypertension. Furthermore, it can lead to compressive symptoms because of compression of the esophagus and airway by the enlarged posterior wall of the LA resulting in dysphagia and respiratory dysfunction [1,6]. Despite all the complications, in rare instances, patients may be completely asymptomatic [7]. The chest radiography is a useful modality for diagnosing LA enlargement. However, it may be difficult to interpret when the left atrium reaches massive proportions. In our case, the chest radiography did not visualize the enlarged cardiac chamber but was revealed by the echocardiogram. It is important to note that the size of the LA does not change after mitral valve replacement (MVR) because of irreversible changes in the atrial muscles by fibrosis [6]. Symptomatic giant LA can be repaired by atrial plication at the time of MVR [8]. However, this procedure can lead to various complications like circumflex coronary artery injury, pulmonary vein obstruction, and esophageal stricture formation [9]. Our case was unusual because the giant LA distorted the cardiac structures with striking cardiomegaly on the chest radiograph and it clouded our interpretation of the cause of the enlarged cardiac silhouette; however, echocardiogram was very helpful in demonstrating the severe MS and the giant LA.

\section{CONCLUSION}

Progressive LA enlargement may be undetected and well-tolerated for a long time, even when it reaches massive proportions. When the LA enlarges to a gigantic size, a chest radiography is not sufficient to delineate the cause of cardiomegaly and can even be misleading. Echocardiogram is the most useful method for evaluating these patients. There is a poor correlation between the size of the LA and the severity of the MS, and our case serves to create awareness to clinicians to consider full diagnostic work up for enlarged LA as patients may not always have symptoms.

\section{ACKNOWLEDGEMENTS}

We thank the patient her family for their valuable support in the preparation of this manuscript.

\section{DECLARATION OF CONFLICT OF INTERESTS}

The abstract of this case has been presented and published in Annual Chest 21 conference and its supplement with DOI: https://doi.org/10.1016/j.chest.2021.07.128

\section{AUTHORS CONTRIBUTIONS}

SV, AP, PS, VJ wrote the abstract, introduction, case, discussion, conclusion. NBP, DS performed critical edits of the draft, did final revision and reshaped it into this manuscript; all authors approved the final version of the manuscript and agree to be accountable for all aspects of the work in ensuring that questions related to the accuracy or integrity of any part of the work are appropriately investigated and resolved.

\section{ETHICS APPROVAL}

Need for ethical approval waived. Consent from the patient deemed to be enough..

\section{CONSENT FOR PUBLICATION}


Written informed consent was obtained from the patient's guardians for publication of this case report and any accompanying images. A copy of the written consent is available for review by the Editor-in-Chief of this journal.

\section{FUNDING}

The authors have not declared a specific grant for this research from any funding agency in the public, commercial or not-for-profit sectors.

\section{REFERENCES}

1. Kawazoe K, Beppu S, Takahara Y, Nakajima N, Tanaka K, Ichihashi K, Fujita T, Manabe H. Surgical treatment of giant left atrium combined with mitral valvular disease: Plication procedure for reduction of compression to the left ventricle, bronchus, and pulmonary parenchyma. The Journal of thoracic and cardiovascular surgery. 1983 Jun 1;85(6):885-92.

2. Hurst JW. Memories of patients with a giant left atrium. Circulation. 2001 Nov 27;104(22):2630-1.

3. Plaschkes J, Borman JB, Merin G, Milwidsky H. Giant left atrium in rheumatic heart disease: a report of 18 cases treated by mitral valve replacement. Annals of surgery. 1971 Aug;174(2):194.

4. Schwammenthal E, Vered Z, Agranat O, Kaplinsky E, Rabinowitz B, Feinberg MS. Impact of atrioventricular compliance on pulmonary artery pressure in mitral stenosis: an exercise echocardiographic study. Circulation. 2000 Nov 7;102(19):2378-84.

5 . Sanfilippo AJ, Abascal VM, Sheehan M, Oertel LB, Harrigan P, Hughes RA, Weyman AE. Atrial enlargement as a consequence of atrial fibrillation. A prospective echocardiographic study. Circulation. 1990 Sep;82(3):792-7.

6 . Apostolakis E, Shuhaiber JH. The surgical management of giant left atrium. European journal of cardio-thoracic surgery. 2008 Feb 1;33(2):182-90.

7. Funk M, Perez M, Santana O. Asymptomatic giant left atrium. Clinical cardiology. 2010 Jun;33(6):E1045 .

8. Isomura T, Hisatomi K, Hirano A, Maruyama H, Kosuga K, Ohishi K. Left atrial plication and mitral valve replacement for giant left atrium accompanying mitral lesion. Journal of cardiac surgery. 1993 May;8(3):36570.

9. Tonguç E, Kestelli M, Özsöyler İ, Yilik L, Yilmaz A, Özbek C, Akçay A, Yeşil M. Limit of indication for plication of giant left atrium. Asian Cardiovascular and Thoracic Annals. 2001 Mar;9(1):24-6.

Table 1 . Causes of left atrial enlargement

\begin{tabular}{l}
\hline Rheumatic Heart Disease \\
\hline Mitral Valve Prolapse \\
Rupture Of Chordae Tendinae \\
Infective Endocarditis \\
Connective Tissue Disorders \\
Calcified Mitral Ring \\
Pericardial Defect \\
Trauma \\
\hline
\end{tabular}



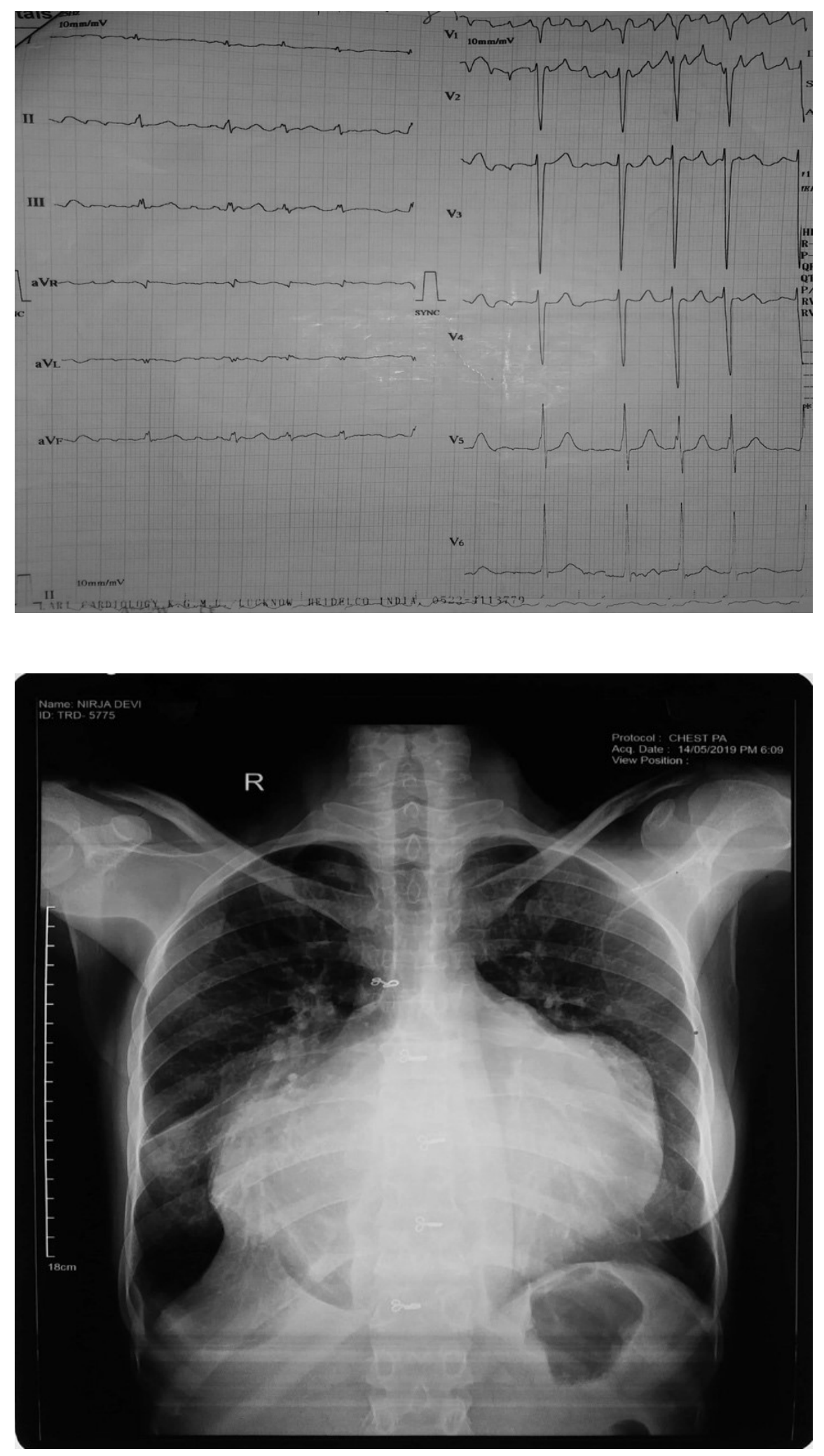


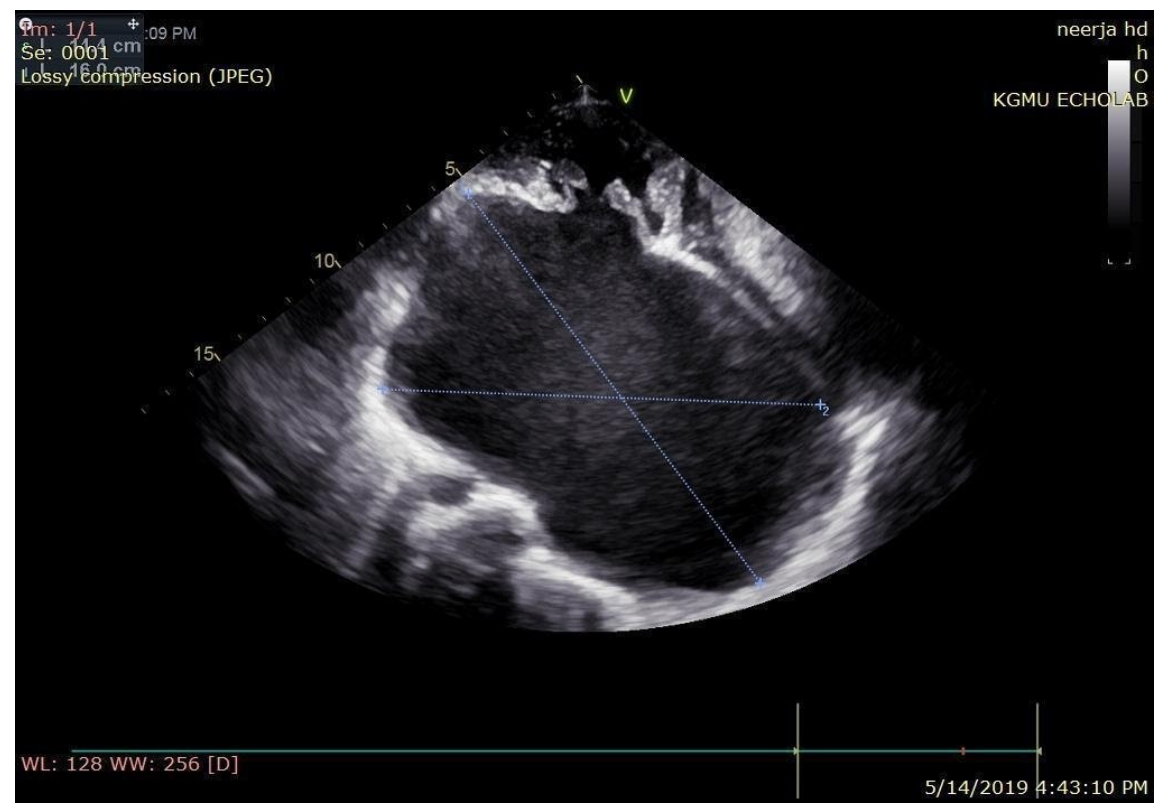

